\title{
Influence of Coulombic Interaction on the
}

\section{Interfacial Self-Assembly of Discotic Liquid}

\section{Crystal Amphiphile: A Combined}

\section{Experimental and Computer Simulation Study}

\author{
Prakhar Verma, ${ }^{\dagger}$ Shakkira Erimban,${ }^{\ddagger}$ Nishant Kumar ${ }^{\dagger}$ Snehasis \\ Daschakraborty, ${ }^{*, \dagger}$ Alpana Nayak, ${ }^{*, \dagger}$ and Sandeep Kumar \\ $\dagger$ †epartment of Physics, Indian Institute of Technology Patna, Patna 801106, India \\ $\ddagger$ Department of Chemistry, Indian Institute of Technology Patna, Patna 801106, India \\ \Raman Research Institute, Sadashivanagar, Bengaluru 560080, India \\ E-mail: snehasis@iitp.ac.in; anayak@iitp.ac.in
}

Supporting Information 
Table 1: Calculated values of surface tensions $(\mathrm{mN} / \mathrm{m})$ and surface pressures $(\mathrm{mN} / \mathrm{m})$ at different surface concentrations $\left(\mathrm{nm}^{2} /\right.$ molecule) for the neutral (sl. no. 1-4) and ionic (sl. no. 5-8) ImTp systems.

\begin{tabular}{llll}
\hline Sl. no. & surface conc. & surface tension & surface pressure \\
\hline 1. & 0.7 & 48.62 & 14.98 \\
2. & 1.0 & 59.62 & 3.98 \\
3. & 1.5 & 58.07 & 5.53 \\
4. & 2.0 & 56.42 & 7.18 \\
\hline \hline 5. & 0.75 & 52.11 & 11.49 \\
6. & 1.0 & 55.1 & 8.5 \\
7. & 2.0 & 57.02 & 6.58 \\
8. & 3.0 & 56.5 & 7.1 \\
\hline
\end{tabular}


(a)

(b)

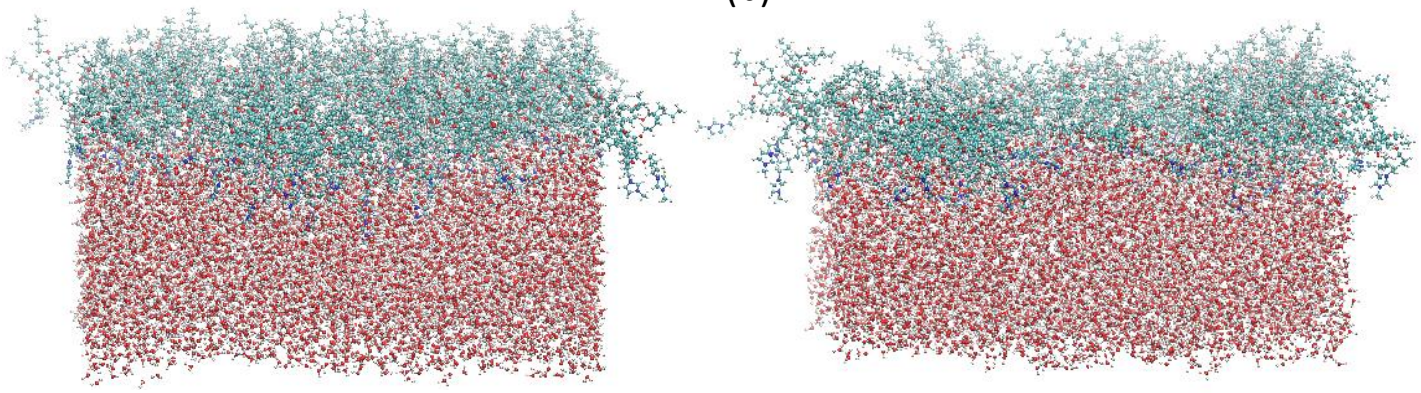

(c)

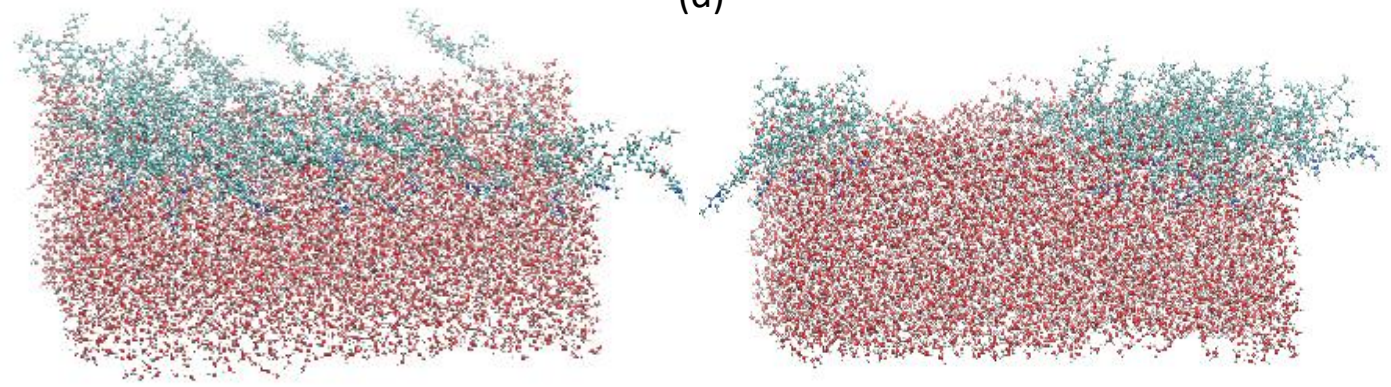

(d)

(e)

(f)

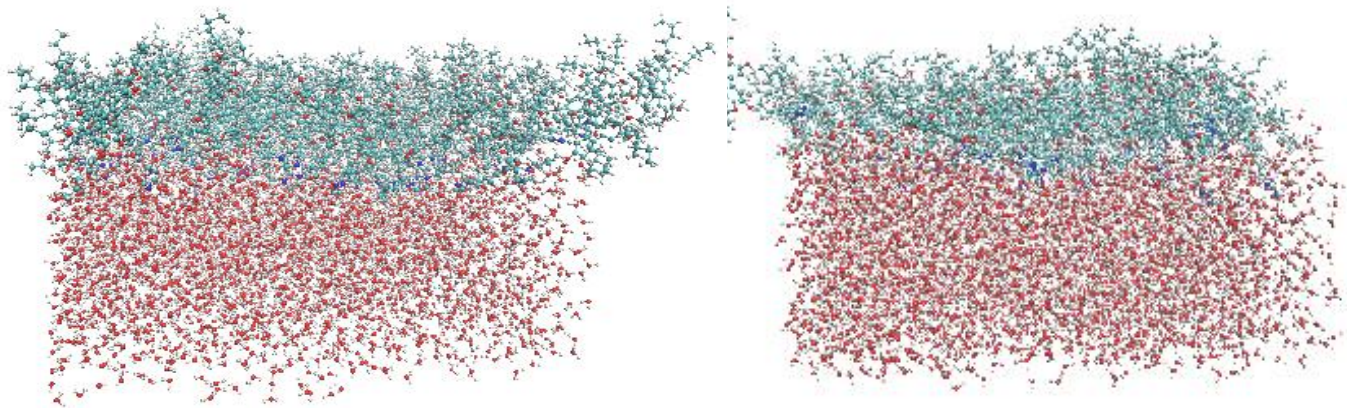

(g)

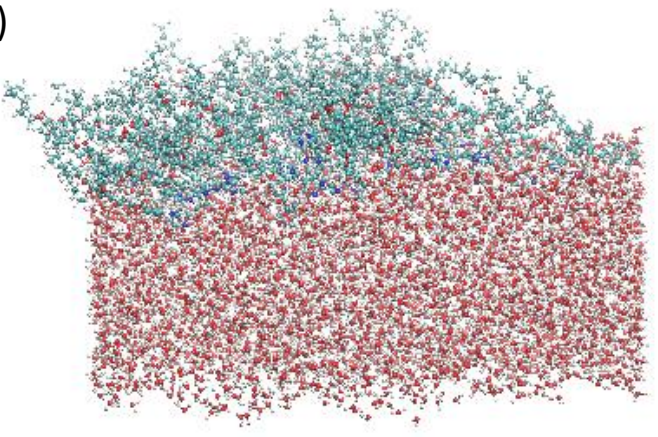

(h)

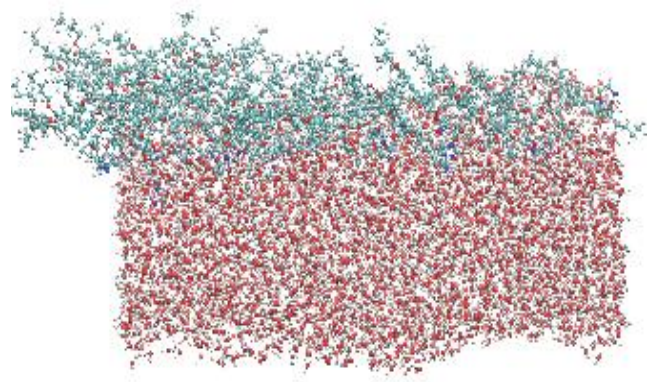

Figure 1: (S1) - Snapshots of the monolayers at $300 \mathrm{~K}$ are shown for (a) $0.7 \mathrm{~nm}^{2}$ (b) $1 \mathrm{~nm}^{2}$ (c) $1.5 \mathrm{~nm}^{2}$ (d) $2 \mathrm{~nm}^{2}$ for neutral-ImTp molecules, and (e) $0.75 \mathrm{~nm}^{2}$ (f) $1 \mathrm{~nm}^{2}$ (g) $2 \mathrm{~nm}^{2}$ (h) $3 \mathrm{~nm}^{2}$ for ionic-ImTp molecules after $30 \mathrm{~ns}$ simulation run. As seen in the Z density profiles of Figure 8 in the main text, an upright alignment is present in the case of smaller surface area and the molecules get aligned to the water surface with increase in the surface area. 


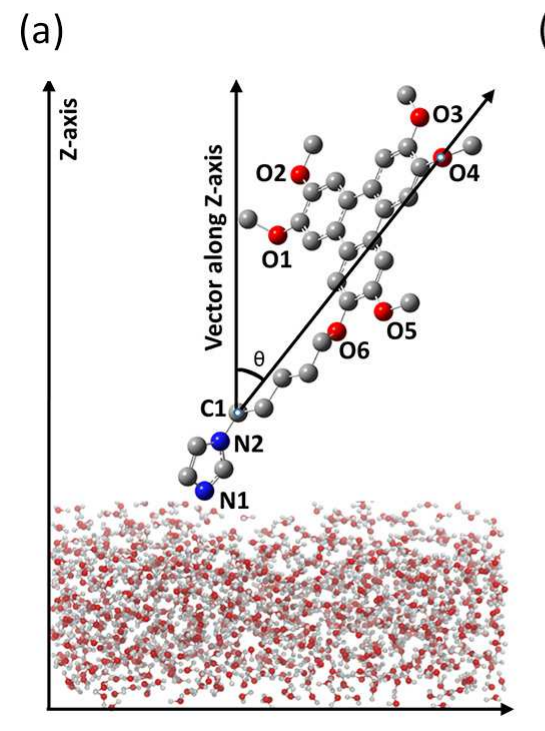

(b)
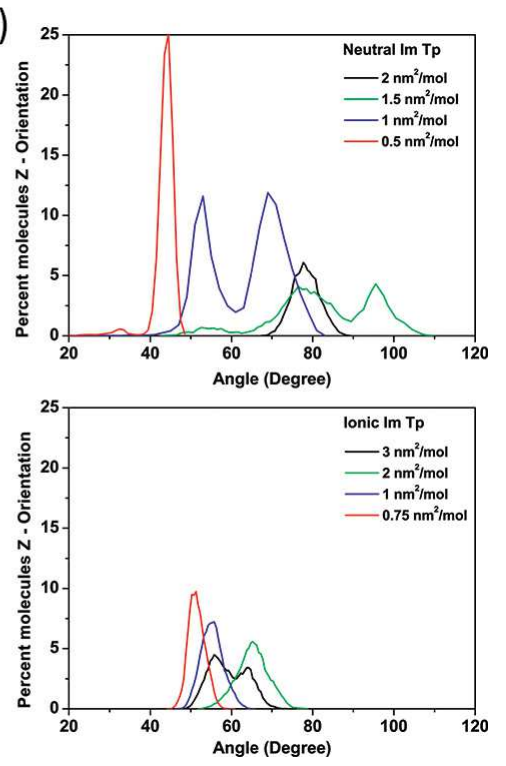

Figure 2: (S2) - (a) The vector considered for Z-orientation along with various labelled atoms for the simplified system with reduced carbon chains to methyl groups only. (b) The percentage of orientation of ImTp molecules as a function of the angle with Z-axis. It is observed that the trend remains the same as in the case of simulations with complete molecules shown in Figure 7 of the main text. 
(a)
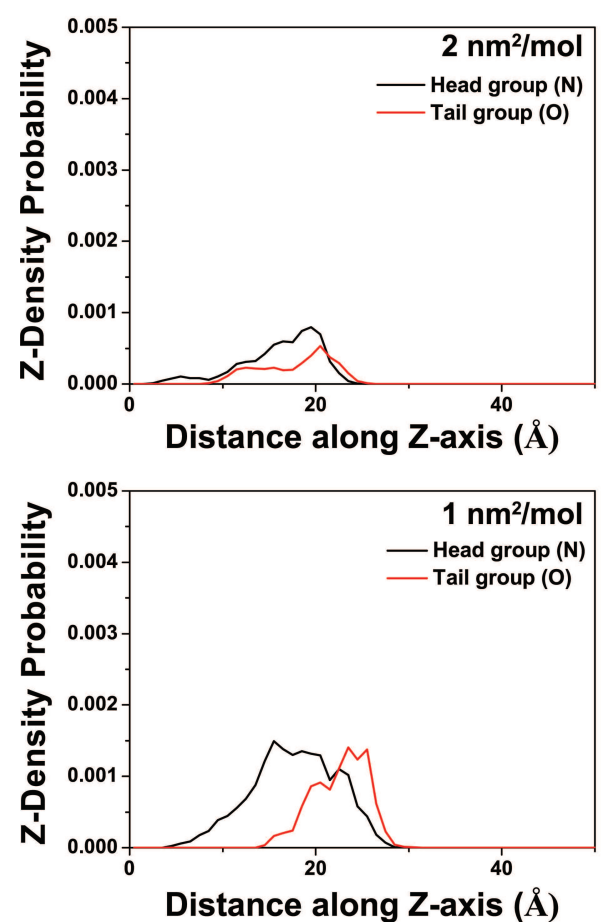

(b)
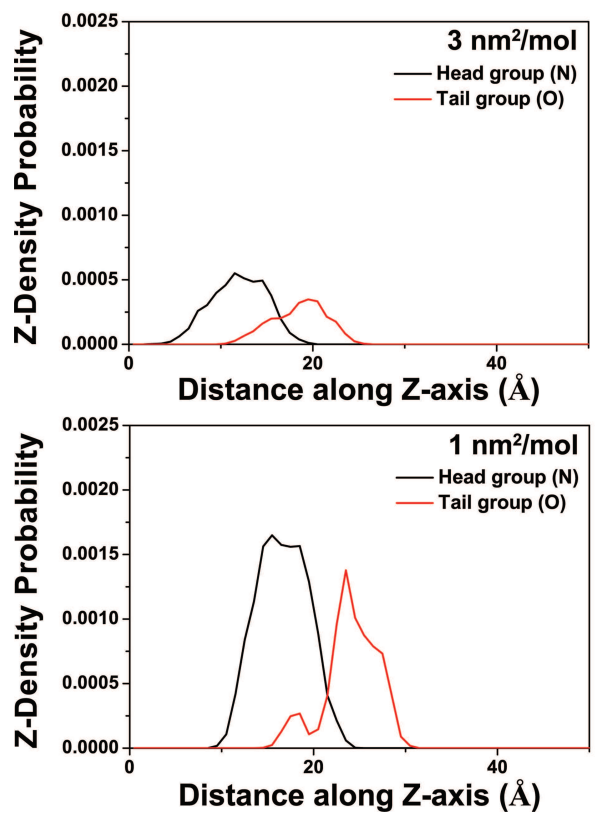

Neutral Im Tp
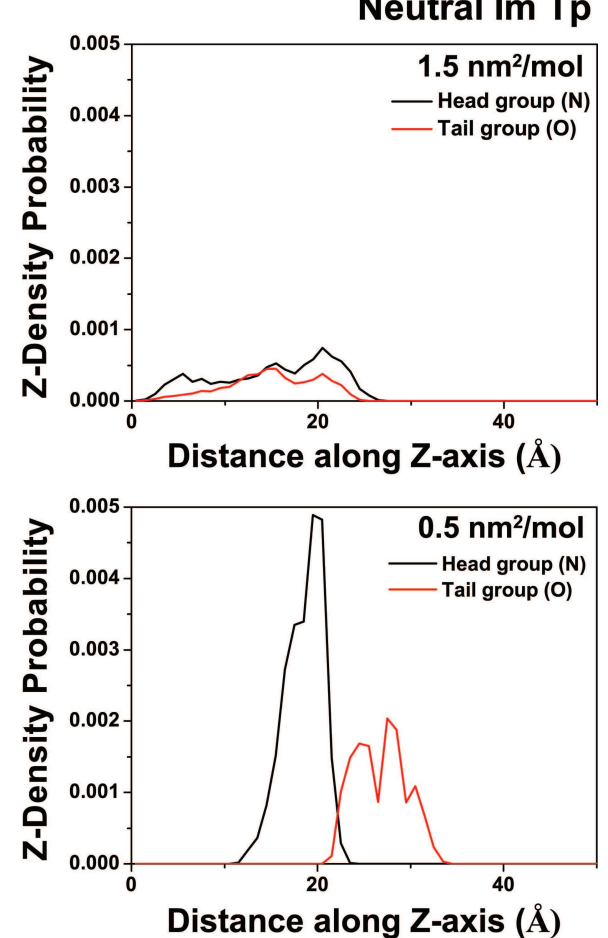

Ionic Im Tp

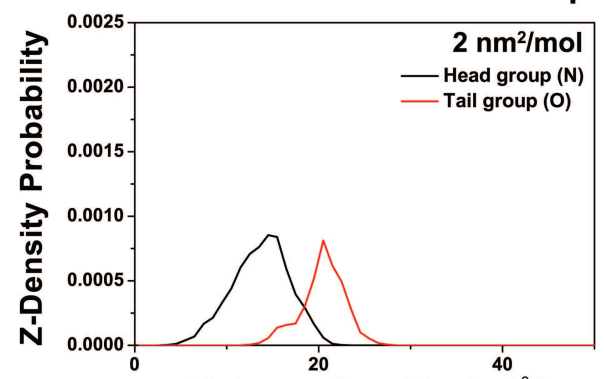

Distance along Z-axis ( $\AA$ )

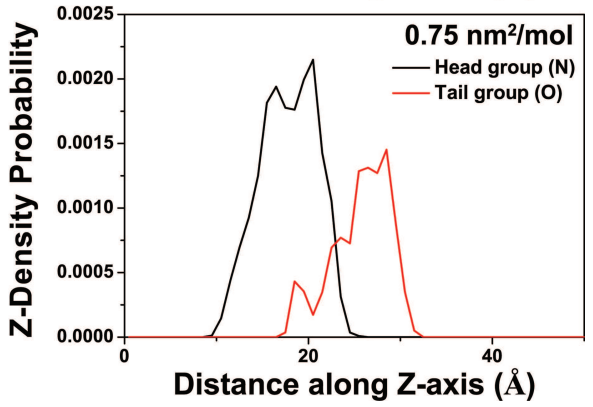

Figure 3: (S3) - For the simplified systems with reduced carbon chains, Z-density probabilities for (a) neutral-ImTp, and (b) ionic-ImTp molecules as surface area varies with respect to the distance along z-axis. Similar to the simulation results for complete molecules as shown in Figure 8 of the main text, the separation of the peaks were higher for neutral molecules than for ionic molecules. Higher the separation, closer is the packing of these molecules. 


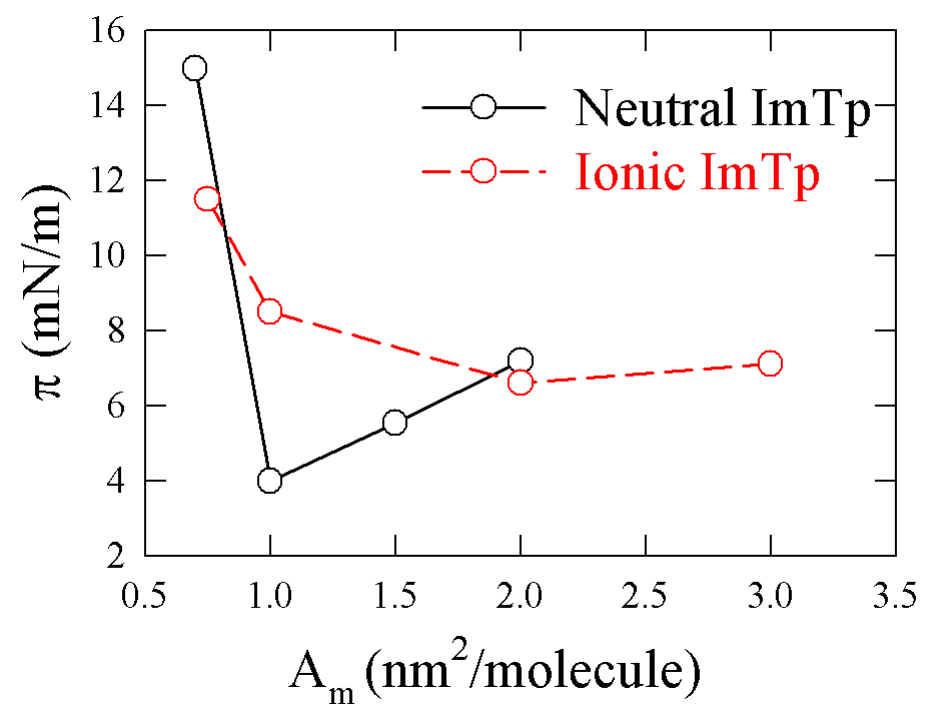

Figure 4: (S4) - Surface pressure vs area isotherm of neutral and ionic ImTp systems predicted qualitatively from simulations. As can be seen, for the neutral system, the surface pressure first decreased and then increased with decreasing surface area. For the ionic system, the surface pressure increased with decreasing surface area as expected. These results did not vary much even for different cut-off values of L-J interactions. It seems that proper choice of force field parameters and right combination of the water models are required to predict the isotherm quantitatively. 